\title{
Análisis de los principales sistemas biológicos de tratamiento de aguas residuales domésticas en Colombia
}

\author{
Biological system analysis for domestic wastewater treatment in Colombia \\ Adriana K.N. Vargas ${ }^{1 *} \quad$ Jimmy Calderón $^{1} \quad$ David Velásquez $^{1}$ \\ Milton Castro $^{1}$ Diego A. Núñez ${ }^{1}$
}

Recibido 23 de noviembre de 2017, aceptado 31 de enero de 2019

Received: November 23, 2017 Accepted: January 31, 2019

\begin{abstract}
RESUMEN
Algunos países en vía de desarrollo no cuentan con sistemas eficientes de tratamiento de aguas residuales; ya sea por problemas económicos, o debido al limitado conocimiento sobre alternativas viables de bajo costo, a pesar de ser una herramienta para mejorar la calidad del agua. Este documento presenta una revisión bibliográfica mediante la cual integra, examina y analiza los diferentes sistemas de tratamiento de agua residual doméstica empleados en Colombia; con el objeto de identificar la evolución que han tenido estas tecnologías y sus posibles alternativas; identificando sus procesos de remoción, costos y la factibilidad en cuanto a la operación. Dentro del análisis, se evidencia que, en Colombia, los tratamientos más usados son los lodos activados, lagunas de estabilización y humedales artificiales. La mayoría de estos tratamientos biológicos alcanzan un $80 \%$ de remoción de $\mathrm{DBO}_{5}$, DQO y SST. Cuando se emplean en conjunto, obtienen eficiencias porcentuales mayores al $90 \%$, resaltando la viabilidad económica de los procesos biológicos, los cuales muestran una mejor rentabilidad económica, funcional y operacional. Sumado a esto, se muestra la necesidad de mejorar los sistemas de tratamiento de aguas residuales existentes en el país, a fin de minimizar la contaminación en aguas superficiales y subterráneas; ya que solo se está interviniendo alrededor del 30\% de las aguas residuales totales, generadas o recibidas por los sistemas de alcantarillado.
\end{abstract}

Palabras clave: Aguas residuales domésticas: tratamiento biológico; eficiencia de remoción.

\begin{abstract}
Some developing countries do not have efficient wastewater treatment systems, either because of economic problems or limited knowledge of viable low-cost alternatives despite it is an option to improve water quality. This document presents a review, which analyzes different wastewater treatment plants in Colombia. The aim is to identify the evolution of these technologies and possible alternatives, processes of removal, costs, and feasibility regarding the operation. The analysis shows that in Colombia, the common treatments are activated sludge, stabilization ponds, and artificial wetlands. Most of these treatments achieve 80\% removal of Biochemical Oxygen Demand, Chemical Oxygen Demand, and Suspended Solids. If these procedures are used together, the percentage of efficiencies will be higher than $90 \%$, highlighting the economic viability of biological processes. Additionally, the study evidences the relevance of improving wastewater treatment systems to minimize pollution in surface and groundwater, considering that only about $30 \%$ of the total wastewater generated or received by the sewage systems is being intervened.
\end{abstract}

Keywords: Domestic wastewater, wastewater treatment systems, biological systems, efficiency.

1 Universidad Manuela Beltrán. 110231. Bogotá D.C., Colombia. E-mail: adriana.nino@ docentes.umb.edu.co; jsebastian3004@ hotmail.com; david.velazquez@umb.edu.co; milton.castro@umb.edu.co; u7700100@unimilitar.edu.co

* Autor de correspondência: adriana.nino@docentes.umb.edu.co 


\section{INTRODUCCIÓN}

Las plantas de tratamiento de aguas residuales en Colombia son una alternativa de final de tubo para el control de la contaminación. Según lo establecido en la normatividad colombiana, la calidad de un efluente doméstico debe cumplir con la Resolución 631 del 2015 del Ministerio de Ambiente y Desarrollo Sostenible, al verterse en una fuente superficial [1].

Los procesos de tratamiento pueden ser físicos, químicos o biológicos [2-3]. En los físicos, se emplea la diferencia entre las propiedades de las partículas con respecto al agua, aplicando principios de separación como la sedimentación o flotación [4]. En los procesos químicos, se utilizan productos como coagulantes o floculantes con el fin de formar partículas coloidales con mayor densidad que luego puedan ser separadas $[6,7]$. En cuanto a los procesos biológicos; los cuales son de mayor aplicación en Latinoamérica para el tratamiento de aguas residuales domésticas (ARD), parten de los principios naturales que ocurren en ríos, lagos o suelos, donde los microorganismos presentes, consumen la materia orgánica (MO), generando material celular; con el objeto de estabilizar, coagular y remover los sólidos coloidales que no se sedimentan de manera natural $[8,9,10,11]$.

El tratamiento de aguas residuales domésticas es empleado para minimizar la contaminación en aguas superficiales y subterráneas [12-13]; dado que, con frecuencia, su disposición final es de manera directa, tanto en cuerpos de agua, como en suelo [14]. Los países en vía de desarrollo presentan bajos porcentajes de agua residual tratada, con respecto a la total generada [15-16] [17], debido, principalmente a razones económicas; a la poca exigencia de las autoridades ambientales y al desconocimiento de tecnologías empleadas para este tipo de procesos [18].

Colombia, es un país caracterizado por su variedad de fuentes hídricas [19], las cuales han sido afectadas de manera paulatina por vertimientos que no consideran el impacto o las consecuencias ambientales producidas [20]. Esto no solo altera negativamente al ecosistema, sino que indirectamente a la población con problemas de salud pública, bienestar y carencia del recurso hídrico de excelente calidad [21-22]. Al año 2011, se había intervenido alrededor del $30 \%$ de las aguas residuales totales, generadas o recibidas por el alcantarillado [23]; sin embargo, el $70 \%$ restante se vierte sin ningún control; observando así, un déficit en cuanto a la eficiencia y aplicación en las Plantas de Tratamiento de Aguas Residuales (PTARs). Sumado a esto, la mayoría de PTARs, no presentan planificación en cuanto al crecimiento poblacional; por lo que, en el transcurso del tiempo, se tornan obsoletas [24, 14].

Por lo anterior, se evidencia la necesidad de evaluar los sistemas de tratamiento de agua residual doméstica, así como la eficiencia de remoción de contaminantes y las alternativas de mejoramiento. Este artículo presenta una revisión bibliográfica, mostrando alternativas viables tanto económicas como eficientes para los sistemas de tratamiento de ARD en Colombia.

\section{Sistemas de aguas residuales en Colombia}

En Colombia se han desarrollado estudios tendientes a evaluar la eficiencia en cuanto a la remoción de contaminantes en sistemas de tratamiento biológico [24-25]. No hay tendencia en la selección del tratamiento, por lo que pueden encontrarse sistemas aerobios, anaerobios, facultativos o la combinación de estos. Las lagunas de estabilización son un claro ejemplo, ya que son empleadas en la mayoría de los municipios del departamento de Cundinamarca. Las PTARs de Gachancipá, Suesca, Tocancipá y Zipaquirá cuentan con estos sistemas lagunares; los cuales han logrado la remoción de hasta el 80\% de la demanda bioquímica de oxígeno $\left(\mathrm{DBO}_{5}\right)$, y una superior al $80 \%$ en cuanto a los sólidos suspendidos totales (SST) [26].

En Santa Fe de Antioquia, departamento de Antioquia, la PTAR cuenta con una laguna anaerobia, conectada en serie con dos lagunas facultativas, operando en paralelo. Según Correa et al. [26], la laguna anaerobia presenta remociones de DBO entre 59\% al $60 \%$, mientras que las lagunas facultativas alcanzan un porcentaje entre $50 \%$ y $70 \%$ cada una; sin embargo, en el análisis del sistema completo, la eficiencia de remoción alcanza un $90 \%$, dando cumplimiento a los valores máximos permitidos por la normatividad ambiental [26].

De acuerdo con lo mencionado por Oswald [27], las lagunas de estabilización deben ser parte fundamental para el manejo de aguas residuales, ya que además de su eficaz proceso en la remoción de 
organismos patógenos, pueden ser implementadas de manera exitosa para la cría de peces y el riego de cultivos. Contribuyendo de manera efectiva a la conservación del recurso hídrico y cuidado del medio ambiente. Por ello, Sanctisa, Moroa, Chimientia, Ritellia, Levantesib y Iaconia [28] proporcionaron un esquema simplificado para el tratamiento y reutilización agrícola de ARD en Italia; basado en lotes secuenciales de sistemas lagunares (SBBGRs); logrando remociones de $90 \%$ de SST y DQO, y el $80 \%$ y $60 \%$ de NT y PT respectivamente. Este sistema también es eficaz en la eliminación de microorganismos (C. perfringens o E. coli) y la calidad del efluente se muestra compatible con los criterios de calidad del agua de la Organización Mundial de la Salud (OMS) para diferentes formas de reúso [29].

En cuanto a sistemas de lagunas algales, Cerón, Madera y Peña [30] examinaron la pertinencia de esta tecnología no convencional en climas tropicales, como se presentan en Colombia; concluyendo que se podría tener excelentes beneficios económicos, al tratar los afluentes contaminados y al mismo tiempo aprovechar la biomasa algal generada; ya sea en la producción de biocombustibles, alimentos, obtención de productos farmacéuticos y la bioprospección de las algas. Sin embargo, la mayoría de las implementaciones se han realizado en zonas con estacionalidad diferenciadas, lo cual podría generar afectación en los resultados esperados [31].

Por otro lado, Arias y Brown [32] cuantificaron el rendimiento y la sostenibilidad de los humedales para el tratamiento de ARD de la sabana de Bogotá, comparándolos con el sistema de lagunas de estabilización y reactor discontinuo secuencial; cada uno utilizando un sistema de rejillas y sedimentación primaria. En el análisis de los tres sistemas de tratamiento, sugieren que el coste anual neto del humedal es de US \$14.672, en comparación con los US \$ 14.201 para las lagunas de estabilización y US \$ 54.887 para el reactor discontinuo. En este orden de ideas, Arias y Brown [32] exponen en términos económicos que el sistema convencional de tratamiento de aguas residuales como lodos activados es entre 2 y 3 veces más costoso que las tecnologías basadas en la naturaleza. Enfatizando que el sistema de lagunas algales es la alternativa más adecuada desde un punto de vista económico.
Sin embargo, el tratamiento de aguas residuales requiere del consumo de una gran cantidad de energía, e incluso este es a menudo el principal costo de operación de los sistemas de tratamiento [33]. Es así como Li, Li y Qiu, [34] investigan el consumo medio de energía en el tratamiento ARD en Shenzhen, China, analizando el costo de energía por unidad tratada; evidenciado que los costos laborales y el consumo de electricidad, corresponden alrededor del 30,1 y el 26,3\% respectivamente, del coste económico total en las PTARs.

Otros sistemas de tratamientos biológicos de aguas residuales en Colombia son los lodos activados [35]; los cuales ayudan en la degradación de la MO [36]. En algunos casos, se han implementado sistemas de clarificación primaria para que las partículas en suspensión caigan y no afecten la eficiencia de remoción [37]. No obstante, un sistema de lodos activados en la modalidad de estabilización por contacto, a escala piloto, elaborado por Mañunga, Rodríguez y Torres [37], evidenció que la clarificación primaria, no genera afectación significativa en la eliminación de carga contaminante. En el estudio realizado, el sistema de lodos activados mostró remociones de 76\%, 80\% y $80 \%$ de $\mathrm{DQO}, \mathrm{DBO}_{5}$ y SST, respectivamente, para ARD en la ciudad de Cali. En esta misma ciudad, Torres, Vásquez, Pérez, Madera y Rodríguez [38] evaluaron 5 sistemas biológicos aerobios a escala de laboratorio para ARD. Fueron usados Lodos Activados y Biodiscos, obteniendo eficiencias de remoción superiores al $80 \%$ en parámetros como $\mathrm{DBO}_{5}$, SST y DQO. Concluyendo la importancia de controlar y analizar los tiempos de retención hidráulica (TRH); dado que con un TRH entre 2 a 4 horas, mejora el desempeño del sistema de Biodiscos, y para un tiempo de operación entre 16 a 12 horas se alcanzan eficiencias hasta de un $90 \%$ [38].

Sobre residuos generados por estas plantas, Cano, Gallego, Velásqueza y Ruiz [39] evaluaron la gestión de biosólidos; identificando procesos sostenibles que logran beneficios económicos con un impacto ambiental mínimo; y un aprovechamiento energético a partir de la gasificación de biosólidos, la cual es energéticamente rentable, económicamente viable y ambientalmente adecuada. Por otra parte, muestran que el uso actual de biosólidos como fertilizante para el suelo no genera ningún tipo de estrés ambiental 
considerable, siempre y cuando se logren eficiencias adecuada; evidenciando el potencial para lograr más beneficios económicos.

Con respecto a los sistemas anaerobios, estos son usados principalmente para pequeñas poblaciones, cuyo principal problema es el poco estudio técnico para su implementación. Este es el caso de un Reactor Anaerobio de Flujo Ascendente (RAFA) implementado en la ciudad de Cali para tratar las aguas de 20000 habitantes donde la producción de olores generó el rechazo de la comunidad [40]. Actualmente, los estudios muestran que, bajo mejoras técnicas, es posible tener una mayor aceptación de los procesos anaerobios con altos porcentajes de remoción. Orozco, Triviño y Manrique [17] estudiaron un RAFA para tratar el ARD en condiciones Andino Amazónicas; obteniendo porcentajes de remoción de DQO mayores al $80 \%$, al alimentar el sistema con una concentración de DQO alrededor de 376 $\mathrm{mg} / \mathrm{L}$, a un caudal de $207 \mathrm{ml} / \mathrm{h}$, mostrando resultados adecuados con remociones mayores al $70 \%$ de MO y un 24\% de coliformes fecales; viabilizando el modelo RAFA, para ser implementado bajo condiciones tropicales [17]. Por otro lado, Stazi y Tomei [41] presentaron remociones de $70 \%$ de DBO para aguas generadas en una población de 320000 habitantes.

Normalmente en Colombia se evalúa la eficiencia de remoción de contaminantes, teniendo en cuenta únicamente parámetros como: $\mathrm{DBO}_{5}$, DQO y SST; sin embargo, el análisis de la remoción de PT y NT es importante para controlar vertimientos a fuentes superficiales con riesgo de eutrofización y baja concentraciones de oxígeno disuelto (OD) [42]; [43]. Para este caso en particular, Gonzalez y Saldarriaga [44] analizaron un tren de tratamiento a escala de laboratorio, compuesto por 3 cámaras: una anaerobia, otra en estado de anoxia y una aerobia; evidenciando una eficiencia de remoción de hasta $92.5 \%$ de MO, $87.7 \%$ en NT y $83.5 \%$ en PT [44]. Este tipo de sistemas resulta ser eficiente para la eliminación simultánea de MO y nutrientes, siendo una opción viable para el tratamiento de ARD a gran escala. Delgadillo, Lopes, Taidi y Pareau [45] presentan altos porcentajes de remoción de nutrientes usando una combinación de procesos físico-químicos y biológicos mediante una laguna algal. Siendo una alternativa atractiva para ser usada en el país.
Los estudios muestran que al implementar solucionas principalmente biológicas, se genera bajo costo de inversión, facilidad de operación y control y la capacidad para amortiguar importantes fluctuaciones de carga orgánica; convirtiéndolo en sistemas con alta rentabilidad a nivel mundial. Esto último, es particularmente útil en el caso de presentarse procesos discontinuos y producciones variables de ARD a lo largo del año.

\section{Comparación con sistemas de tratamiento en el mundo}

Las exigencias normativas en otras partes del mundo, así como la necesidad de un aprovechamiento del vertimiento generado, hacen que se obtengan mayores eficiencias de remoción de contaminantes usando sistemas biológicos. En países como España, se muestran mejores eficiencias en cuanto a sistemas lagunares. Martínez, García Martínez, Medina y Almela [46] evaluaron el rendimiento de un humedal artificial de flujo subsuperficial horizontal (HF-CW), artificialmente aireado; el cual fue diseñado para el tratamiento de las ARD, teniendo como objeto producir efluentes adecuados para la reutilización del recurso. Obteniendo remociones de turbidez, sólidos suspendidos totales (SST), nitrógeno total (TN), Escherichia coli (E. coli) y fósforo total (TP), con una reducción media de 96,9\%. Cabe resaltar que este es un sistema no convencional y poco empleado en Colombia [47].

Con respecto a la remoción de nutrientes, Hülsen, Barry, Lu, Puyol, Keller y Batstone, [48] propusieron un sistema biorreactor de membrana continua foto-anaeróbico, en el cual se emplean bacterias fototróficas, las cuales tienen el potencial biológico de almacenar nutrientes de acuerdo con su crecimiento. Sin embargo, como tecnología nueva presenta desafíos con altas cargas contaminantes y aún no ha sido comprobada su eficiencia a gran escala. No obstante, permite evidenciar que es una alternativa factible para un desarrollo sostenible. Omole, Ndambuki y Kupolati [49] examinan un sistema secuencial de lodos activados, combinado con una vegetación de cama de humedales artificiales sumergidos; para la eliminación ARD, mostrando un porcentaje de eliminación de 92,48\% de NT; $88,78 \%$ de sulfatos (S); y $99,6 \%$ de SST. Usando macrófitas disponibles localmente en combinación con especies no nativas, aumentó significativamente la eficiencia en cuanto a este proceso. Stazi y 
Tomei [41] compararon las eficiencias de sistemas UASB en diferentes países tropicales y bioreactores anaerobios de membrana, los cuales se muestran como alternativas promisorias para el tratamiento anaerobio de aguas residuales domésticas donde es posible alcanzar hasta el $99 \%$ en remociones de DQO.

La Tabla 1 indica los porcentajes de eficiencia de remoción de acuerdo con los diferentes autores analizados en esta revisión.

\section{CONCLUSIONES}

Existen otras alternativas tratamiento biológico; pero los más utilizados en el territorio colombiano resultan ser las lagunas de estabilización, humedales y los lodos activados. Según los resultados, se demuestra que los sistemas biológicos presentan altos porcentajes de eficiencia de remoción de contaminantes, pero cuando estos se emplean en conjunto, obtienen eficiencias porcentuales mayores al $90 \%$, evidenciando que estos sistemas complementados, son óptimos para el tratamiento de aguas residuales domésticas.
Pocos estudios han calculado específicamente los costos económicos de los sistemas de tratamiento $\mathrm{ARD}$; por lo que se hace necesario generar un modelo que cuantifique, en términos económicos, el cambio de estado del agua tratada al agua regenerada, favoreciendo la toma de decisiones más eficiente con respecto a este recurso. Sin embargo, como resultado se puede denotar que la reutilización de las aguas residuales es una opción interesante en términos de costos en comparación con otras fuentes de agua existentes: las aguas superficiales, aguas subterráneas o la desalinización. Adicionalmente, cabe resaltar la viabilidad económica de los sistemas que simulan procesos naturales como: lagunas de estabilización y humedales artificiales, los cuales mostraron la mejor rentabilidad económica, funcional y operacional.

En Colombia es necesario avanzar en las técnicas de tratamiento de ARD, para disminuir las concentraciones de contaminación sobre los cuerpos de agua superficial; para ello se debe empezar con una ampliación de los sistemas actuales de tratamiento de este tipo de vertimientos, mediante el montaje de procesos biológicos como los descritos en este

Tabla 1. Resumen de revisión bibliográfica de los porcentajes de eficiencia de remoción de acuerdo a los diferentes autores.

\begin{tabular}{|c|c|c|c|c|c|c|c|}
\hline \multirow{2}{*}{ Autor(es) } & \multirow{2}{*}{ Sistema de tratamiento de ARD } & \multicolumn{6}{|c|}{ Porcentajes de remoción } \\
\hline & & $\mathrm{DBO}_{5}$ & DQO & SST & NT & PT & $S$ \\
\hline $\begin{array}{l}\text { Gonzalez y Saldarriaga } \\
\text { (2008). }\end{array}$ & $\begin{array}{l}\text { Tipo A2O } \\
\text { (anaerobio-anóxico-aerobio). }\end{array}$ & $92,5 \%$ & NA & - & $87,7 \%$ & $83,5 \%$ & - \\
\hline $\begin{array}{l}\text { Méndez, Carreño y } \\
\text { Hernández (2011). }\end{array}$ & Lagunas de estabilización. & $80,0 \%$ & $\leq 80 \%$ & - & - & - & - \\
\hline Torres (2011). & $\begin{array}{l}\text { Lodos activados combinado con } \\
\text { sistema de Biodiscos. }\end{array}$ & $\leq 80 \%$ & $\leq 80 \%$ & $\leq 80 \%$ & - & - & - \\
\hline $\begin{array}{l}\text { Correa, Cuervo, Mejía y } \\
\text { Aguirre (2012). }\end{array}$ & $\begin{array}{l}\text { Laguna anaerobia, conectada en } \\
\text { serie con dos lagunas facultativas, } \\
\text { operando en paralelo. }\end{array}$ & $90,0 \%$ & - & - & - & - & - \\
\hline $\begin{array}{l}\text { Mañunga, Rodríguez y } \\
\text { Torres (2011). }\end{array}$ & Lodos activados. & $80,0 \%$ & $76,0 \%$ & $80,0 \%$ & - & - & - \\
\hline $\begin{array}{l}\text { Orozco, Triviño y Manrique } \\
(2014) .\end{array}$ & $\begin{array}{l}\text { Reactor anaerobio de Flujo } \\
\text { Ascendente. }\end{array}$ & $\leq 70 \%$ & $\leq 80 \%$ & - & - & - & - \\
\hline $\begin{array}{l}\text { Badejo, Omole, Ndambuki y } \\
\text { Kupolati (2017). }\end{array}$ & $\begin{array}{l}\text { Lodos activados, combinado } \\
\text { con una vegetación de cama de } \\
\text { humedales artificiales sumergidos. }\end{array}$ & - & - & $99,6 \%$ & $99,6 \%$ & - & $88,8 \%$ \\
\hline $\begin{array}{l}\text { Martínez, García Martínez, } \\
\text { Medina y Almela (2017). }\end{array}$ & $\begin{array}{l}\text { Humedal artificial de flujo } \\
\text { subsuperficial horizontal. }\end{array}$ & $97,8 \%$ & $92,7 \%$ & $97,5 \%$ & $91,5 \%$ & $96,9 \%$ & - \\
\hline $\begin{array}{l}\text { Sanctisa, Moroa, Chimientia, } \\
\text { Ritellia, Levantesib y Iaconia } \\
\text { (2017). }\end{array}$ & $\begin{array}{l}\text { Lotes secuenciales de sistemas } \\
\text { lagunares. }\end{array}$ & $98,0 \%$ & $90,0 \%$ & $90,0 \%$ & $80,0 \%$ & $60,0 \%$ & - \\
\hline
\end{tabular}


texto. Además, se tienen que planificar PTARs en los municipios o zonas donde no presentan un sistema de tratamiento, las cuales consideren el crecimiento de la población y garanticen una buena eficiencia de remoción de MO y otros contaminantes.

De acuerdo con los estudios realizados por los diferentes autores, los principales sistemas de tratamiento biológicos implementados en Colombia, han mostrado grandes eficiencias de remoción de parámetros como $\mathrm{DBO}_{5}$, DQO, SST y coliformes. Sin embargo, la viabilidad de implementar un sistema biológico determinado, depende de las características del ARD, el caudal a tratar, los TRH y costos de implementación, mantenimiento y operación.

De igual manera, la falta de un sistema de pretratamiento, genera deficiencias en la remoción de contaminantes por parte de los sistemas biológicos, así como, problemas de operación. Por esta razón, se hace necesario implementar sistemas de pretratamiento, los cuales permitan remover todo material flotante de gran tamaño; el uso de sistemas de trampa de grasas para evitar cambios de $\mathrm{pH}$ y un sistema de clarificación primaria para amortiguar las cargas de choque y evitar problemas operacionales por la variación del caudal $[37,50]$.

\section{AGRADECIMIENTOS}

A todos los autores y colaboradores por brindar el marco de desarrollo del presente trabajo.

\section{REFERENCIAS}

[1] República de Colombia. "Resolución 1096". Ministerio de Desarrollo Económico, Bogotá D.C. 2000.

[2] J.A.G. Anaya and R.N. Gutiérrez. "Effect of domestic and coffee mill discharges on the water quality and the Odonata (Insecta) larval diversity in a mountain cloud forest stream in Veracruz, Mexico". Revista Mexicana de Biodiversidad, pp. 372-380. 2017.

[3] J.A. Jácome, J. Molina, J. Suárez, G. Mosqueira and D. Torres. "Performance of constructed wetland applied for domestic wastewater treatment: Case study at Boimorto (Galicia, Spain)”. Ecological Engineering, pp. 324-329. 2016.
[4] P.V.M. Luna y C.S. Aburto. "Sistema de humedales artificiales para el control de la eutrofización del lago del Bosque de San Juan de Aragón”. TIP Revista Especializada en Ciencias Químico-Biológicas, pp. 32-55. 2014.

[5] N. Libardi, C.R. Soccol, A.G. Neto, J.d. Oliveira and L.P.d.S. Vandenberghe. "Domestic wastewater as substrate for cellulase production by Trichoderma harzianum". Process Biochemistry, pp. 190-199. 2017.

[6] E. GilPavasa, I. Dobrosz and M.Á. Gómez. "Coagulation-flocculation sequential with Fenton or Photo-Fenton processes as an alternative for the industrial textile wastewater treatment". Journal of Environmental Management. Vol. 191, pp. 189-197. 15-04-2017.

[7] Y. Zhang, S. Li, X. Wang and X. Li. "Coagulation performance and mechanism of polyaluminum ferric chloride (PAFC) coagulant synthesized using blast furnace dust". Separation and Purification Technology. Vol. 154, pp. 345-350. 05-11- 2015.

[8] P. Torres. "Perspectivas del tratamiento anaerobio de aguas residuales domésticas en países en desarrollo". Escuela de Ingeniería de Antioquia, Medellín (Colombia), pp. 115129. 2012.

[9] M. Fountoulakis, G. Sabathianakis, I. Kritsotakis, E. Kabourakis and T. Manios. "Halophytes as vertical-flow constructed wetland vegetation for domestic wastewater treatment". Science of The Total Environment, pp. 432-439. 01-04-2017.

[10] Y.P.H. Choa, J. Yua, B. Minb, H.S. Kimc. B.G. Kimc and T. Leea. "Response of microbial community structure to preacclimation strategies in microbial fuel cells for domestic wastewater treatment". Bioresource Technology. Vol. 233, pp. 176183. June 2017.

[11] I.V. Muralikrishna and V. Manickam. "Environmental Management". Chapter Twelve - Wastewater Treatment Technologies, pp. 249-293. 2017.

[12] S. Arora and A. Kazmi. "The effect of seasonal temperature on pathogen removal efficacy of vermifilter for wastewater treatment". Water Research. Vol. 74 N $^{\circ} 1$, pp. 88-99. May, 2015. 
[13] C. Prasse, D. Stalter, U. Schulte-Oehlmann, J. Oehlmann and T.A. Ternes. "Spoilt for choice: A critical review on the chemical and biological assessment of current wastewater treatment technologies". Water Research. Vol. 87, pp. 237-270. 15-12-2015.

[14] J. Silva, P. Torres and C. Madera. "Reuso de aguas residuales domésticas en agricultura. Una revisión". Agronomía Colombiana. Vol. XXVI Nº 02, pp. 347-359. 10-07-2008.

[15] R. Martins, C. Quintal, L. Cruz and E. Barata. "Water affordability issues in developed countries - The relevance of micro approaches". Utilities Policy. Vol. 43, pp. 117-123. December, 2016.

[16] S. Mathur. "Public-private partnership for municipal water supply in developing countries: Lessons from Karnataka, India, Urban Water Supply Improvement Project". Cities. Vol. 68, pp. 56-62. August, 2017.

[17] A. Orozco, C. Triviño y L. Manrique. "Arranque de un reactor UASB para el tratamiento de aguas residuales domésticas en condiciones Andino Amazónicas" Universidad Militar Nueva Granada. Vol. X $\mathrm{N}^{\circ}$ 2, pp. 170-185. 05-09-2014.

[18] P. Torres. "Perspectives of anaerobic treatment of domestic wastewater in developing countries". Revista EIA. No 18. pp. 115-129. December, 2012.

[19] L. Londoño, J. Segrera and M. Jaramillo. "Water Distribution System of Santa Marta city, Colombia". Procedia Engineering. Vol. 186, pp. 20-27. 2017.

[20] G.M.P. Salazar. "Water Distribution and Drainage Systems of Aburrá Valley, Colombia Empresas Públicas de Medellín E.S.P.'. Procedia Engineering. Vol. 186, pp. 4-11. 2017.

[21] B.R. Goldman, S. Benitez, A. Calvache, A. Ramos and F. Veiga. "Water-borne protozoa parasites: The Latin American perspective". International Journal of Hygiene and Environmental Health. Vol. $220 \mathrm{~N}^{\circ}$ 5, pp. 783-798. July 2017.

[22] Instituto Colombiano de Salud. "Estado de vigilancia de la calidad de agua para consumo humano en Colombia”. Bogotá D.C. 2015.

[23] Superintendencia de Servicios Públicos. "Informe técnico sobre sistemas de tratamiento de aguas residuales en Colombia”. Bogotá. 2016.
[24] J. Romero. "Tratamiento de aguas residuales". Bogotá: Escuela colombiana de ingeniería, pp. 162-169. 2000.

[25] M.A. Jiménez and F.L. Santana. "Water Distribution System of Bogotá City and Its Surrounding Area, Empresa de Acueducto y Alcantarillado de Bogotá - EAB E.S.P”. Procedia Engineering. Vol. 186, pp. 643-653. 2017.

[26] J. Méndez, F. Carreño y A. Hernández. "Confiabilidad y viabilidad para la reutilización de los efluentes de las Ptar que operan con lagunas de estabilización en cundinamarca”. Producción + Limpia. Vol. VI No 01, pp. 35-49. Junio 2011.

[27] G. Correa, H. Cuervo, R. Mejía y N. Aguirre. "Monitoreo del sistema de lagunas de estabilización del municipio de Santa Fé de Antioquia, Colombia" Producción + Limpia. Vol. VII Nº 02, pp. 36-51. Diciembre 2012.

[28] M.D. Sanctisa, G.D. Moroa, S. Chimientia, P. Ritellia, C. Levantesib and C.D. Iaconia. "Removal of pollutants and pathogens by a simplified treatment scheme for municipal wastewater reuse in agriculture". Science of The Total Environment. Vol. 580, pp. 17-25. 15-02-2017.

[29] G.S. León. "Parámetros de calidad para el uso de aguas residuales. guías de calidad de efluentes para la protección de la salud". Organización Mundial para la Salud. 2016.

[30] V.A. Cerón, C.A. Madera y M. Peña. "Uso de lagunas algales de alta tasa para tratamiento de aguas residuales" Ingeniería. Vol. $33 \mathrm{~N}^{\circ} 1$. Enero 2015.

[31] J. Roostaei and Y. Zhang. "Spatially Explicit Life Cycle Assessment: Opportunities and challenges of wastewater-based algal biofuels in the United States". Algal Research. Vol. 24 No b, pp. 395-402. June 2017.

[32] M. Arias and M. Brown. "Feasibility of using constructed treatment wetlands for municipal wastewater". Ecological Engineering. Vol. XXXV Nº 07, pp. 10701078. March, 2009.

[33] I.R. Rosaa, F.J. García and J. Mendoza. "Development and application of a cost management model for wastewater treatment and reuse processes". Journal of Cleaner Production. Vol. 113, pp. 299-310. 01-02-2016. 
[34] W. Li, L. Li and G. Qiu. "Energy consumption and economic cost of typical wastewater treatment systems in Shenzhen, China". Journal of Cleaner Production. Acceso en línea: 08-01-2016.

[35] Ó. Giraldo and A. Lozano. "Effect of drying of biosolids from the wastewater treatment plant El Salitre (Bogotá) on the content of nutrients, heavy metals, and pathogens". Agronomía Colombiana. Vol. XXII, N ${ }^{\circ} 02$, pp. 348-354. December 2006.

[36] M.R. Espinosa. "Optimización de la Producción de Lodos En un Sistema de lodos activados a Través de la calibración del modelo ASM1". Ingeniería, Investigación y Tecnología. pp. 93-104. Enero 2015.

[37] T. Mañunga, J.A. Rodríguez y P. Torres. "Tratamiento de agua residual doméstica sin clarificación primaria en un sistema de lodos activados en la modalidad de estabilización por contacto". Ingeniería y Desarrollo. vol. XXX, No 02. Diciembre 2012.

[38] P. Torres, N. Vásquez, A. Pérez, C. Madera y J. Rodríguez. "Alternativas de tratamiento biológico aerobio para el agua residual doméstica del municipio de Cali, Colombia". Afinidad, Revista de química teórica y aplicada. Vol. LXVIII, $\mathrm{N}^{\circ} 555$. Noviembre 2011.

[39] N.A. Cano, D. Gallego, H.I. Velásqueza y G.J. Ruiz. "Emergy analysis for the sustainable utilization of biosolids generated in a municipal wastewater treatment plant". Journal of Cleaner Production. Vol. 141. 10-01-2017.

[40] T.J.J. Kalker, J.A.W Maas, R.R. Zaag. "Transfer and acceptance of UASB technology for domestic wastewater: Two cases studies". Water Science Technology. Vol. 39, pp. 219225. May, 1999.

[41] V. Stazi and M.C. Tomei. "Enhancing anaerobic treatment of domestic wastewater: State of the art, innovative technologies and future perspectives". Journal Science of The Total Environment. Vol. 635, pp. 78-91. 2018.

[42] G. Manninaa, M. Capodicia, A. Cosenzaa, D.D. Trapania and M.C.V. Loosdrechtb. "Nitrous oxide emission in a University of
Cape Town membrane bioreactor: The effect of carbon to nitrogen ratio". Journal of Cleaner Production, pp. 180-190. 17-04-2017.

[43] Y.K. Reddya, S.A.b, E. K. Levlina y K. Reddyd. "Enhancing nitrogen removal efficiency of domestic wastewater through increased total efficiency in sewage treatment (ITEST) pilot plant in cold climatic regions of Baltic Sea". International Journal of Sustainable Built Environment. Acceso en línea: 25-05-2017.

[44] M. Gonzalez y J. Saldarriaga. "Remoción biológica de materia orgánica, nitrógeno y fosforo en un sistema tipo anaerobio-anóxicoaerobio". Revista EIA. No 10, pp. 45-53. 2008.

[45] L. Delgadillo, F. Lopes, B. Taidi and D. Pareau. "Nitrogen and phosphate removal from wastewater with a mixed microalgae and bacteria culture". Journal of Biotechnology Reports. Vol. 11, pp. 18-26. September 2016.

[46] P.A. Martínez, N. García Martínez, J.Q. Medina y L. Almela. "Domestic wastewaters reuse reclaimed by an improved horizontal subsurface-flow constructed wetland: A case study in the southeast of Spain". Bioresource Technology, pp. 236-246. 2017.

[47] Ministerio de Vivienda, Ciudad y Territorio. "Decreto 1898". República de Colombia. 11-23-2016.

[48] T. Hülsen, E.M. Barry, Y. Lu, D. Puyol, J. Keller and D.J. Batstone. "Domestic wastewater treatment with purple phototrophic bacteria using a novel continuous photo anaerobic membrane bioreactor". Water Research. Vol. 100, pp. 486-495. 01-09-2016.

[49] A.A. Badejo, D.O. Omole, J.M. Ndambuki and W.K. Kupolati. "Municipal wastewater treatment using sequential activated sludge reactor and vegetated submerged bed constructed wetland planted with Vetiveria zizanioides". Ecological Engineering, pp. 525-529. 2017.

[50] M.Z. Pereyra, S. Lavrnić, F.V. Dien, J.V. Bruggen and P. Lens. "Constructed wetroofs: A novel approach for the treatment and reuse of domestic wastewater". Ecological Engineering, pp. 545-554. 2016. 